\title{
A cost-effectiveness analysis of long-term intermittent catheterisation with hydrophilic and uncoated catheters
}

\author{
JF Clark ${ }^{1}$, SJ Mealing ${ }^{1}$, DA Scott ${ }^{1},{\text { LC } \text { Vogel }^{2}, \text { A Krassioukov }}^{3}$, M Spinelli ${ }^{4}$, P Bagi ${ }^{5}$ and J-J Wyndaele ${ }^{6}$
}

Study design: Cost-effectiveness analysis

Objective: To establish a model to investigate the cost effectiveness for people with spinal cord injury (SCI), from a lifetime perspective, for the usage of two different single-use catheter designs: hydrophilic-coated (HC) and uncoated (UC). The model includes the long-term sequelae of impaired renal function and urinary tract infection (UTI).

Setting: Analysis based on a UK perspective.

Methods: A probabilistic Markov decision model was constructed, to compare lifetime costs and quality-adjusted life years, taking renal and UTI health states into consideration, as well as other catheter-related events. UTI event rates for the primary data set were based on data from hospital settings to ensure controlled and accurate reporting. A sensitivity analysis was applied to evaluate best- and worst-case scenarios.

Results: The model predicts that a 36-year-old SCI patient with chronic urinary retention will live an additional 1.4 years if using $\mathrm{HC}$ catheters compared with UC catheters, at an incremental cost of $£ 2100$. Moreover, the lifetime number of UTI events will be reduced by $16 \%$. All best- and worst-case estimates were within the UK threshold of being cost effective.

Conclusion: The use of $\mathrm{HC}$ catheters for intermittent catheterisation in $\mathrm{SCl}$ patients is highly cost effective. The outcome is consistent irrespective of whether UTI data are collected in hospital or community settings.

Spinal Cord (2016) 54, 73-77; doi:10.1038/sc.2015.117; published online 21 July 2015

\section{INTRODUCTION}

Approximately 40000 people in the UK live with a spinal cord injury (SCI), and the current annual cost of caring for these patients is estimated to be in excess of $\mathfrak{£ 5 0 0}$ million. ${ }^{1}$ The majority of SCI patients suffer from neurogenic bladder disturbances, including chronic urinary retention. ${ }^{2}$ Intermittent catheterisation (IC) is considered the gold standard for long-term bladder management in this patient group, as it reduces catheter-associated urinary tract infections (UTI) and promotes greater independence. The IC procedure is also recommended by the National Institute for Health and Care Excellence (NICE). ${ }^{3}$

IC catheters are designed for either multiple or single use. Multipleuse catheters are uncoated (UC) whereas single-use catheters are either coated or UC. In the UK, single-use catheters are considered the standard method for IC. According to the current European Association of Urology Nurses guidelines, the use of UC catheters are deemed as a factor that increases the risk of IC-related UTIs. The guidelines highlight the use of single-use, hydrophilic-coated (HC) or gelreservoir catheters, which they consider to reduce the risk of urethral trauma-another common complication of IC. ${ }^{4}$ A recently updated Cochrane review examined the evidence on whether catheter design affects the long-term incidence of IC-related UTI. ${ }^{5}$ Overall, there were no conclusive data for the individual catheter types, and future trials including cost-effectiveness analyses are recommended. Bermingham et al. ${ }^{6}$ published a cost-effectiveness analysis, using data from trials with various different IC catheter types. ${ }^{6}$ The analysis focused on the acute treatment of symptomatic UTIs, but did not consider their lifetime downstream sequelae such as renal function. Furthermore, the analysis by Bermingham et al. did not include the most recent and largest study in hospitalised SCI patients comparing UTI incidence rates for single-use $\mathrm{HC}$ and UC catheters. ${ }^{7}$ The objective of the present study was to perform a cost-effectiveness analysis with a lifetime perspective of the use of two different single-use catheter designs in an adult SCI population, including long-term sequelae of impaired renal function and accessing the latest evidence.

\section{MATERIALS AND METHODS}

\section{Design of decision model}

A probabilistic Markov decision model $^{8}$ was constructed to evaluate the cost effectiveness of using HC catheters versus UC catheters. This model was designed to capture the different health states that a catheter-dependant SCI patient may experience throughout his or her lifetime. Health-related quality of life was incorporated into the model in order to capture the patient's well being (see Supplementary Material). An expert panel of six urologists and SCI rehabilitation specialists helped to develop the model structure and assessed the assumptions used. Figure 1 shows an overview of the model. The SCI cohort was classified according to the health state of their renal function (RF): RF-I (no or minor renal impairment), RF-II (major renal impairment, requiring intervention together with careful medical monitoring) and RF-III

${ }^{1}$ ICON Health Economics \& Epidemiology, Oxford, UK; ${ }^{2}$ Shriners Hospitals for Children, Chicago, IL, USA; ${ }^{3}$ Department of Medicine, University of British Columbia, Vancouver, British Columbia, Canada; ${ }^{4}$ Unità Spinale Unipolare, Azienda Ospedale Niguarda Cà Granda, Milan, Italy; ${ }^{5}$ Department of Urology, Rigshospitalet, University of Copenhagen, Copenhagen, Denmark and ${ }^{6}$ University of Antwerp and University Hospital Antwerp, Antwerp, Belgium

Correspondence: SJ Mealing, ICON Clinical Research (UK) Ltd., Seacourt Tower, West Way, Oxford OX2OJJ, UK.

E-mail: Stuart.Mealing@iconplc.com

Received 22 December 2014; revised 29 May 2015; accepted 9 June 2015; published online 21 July 2015 


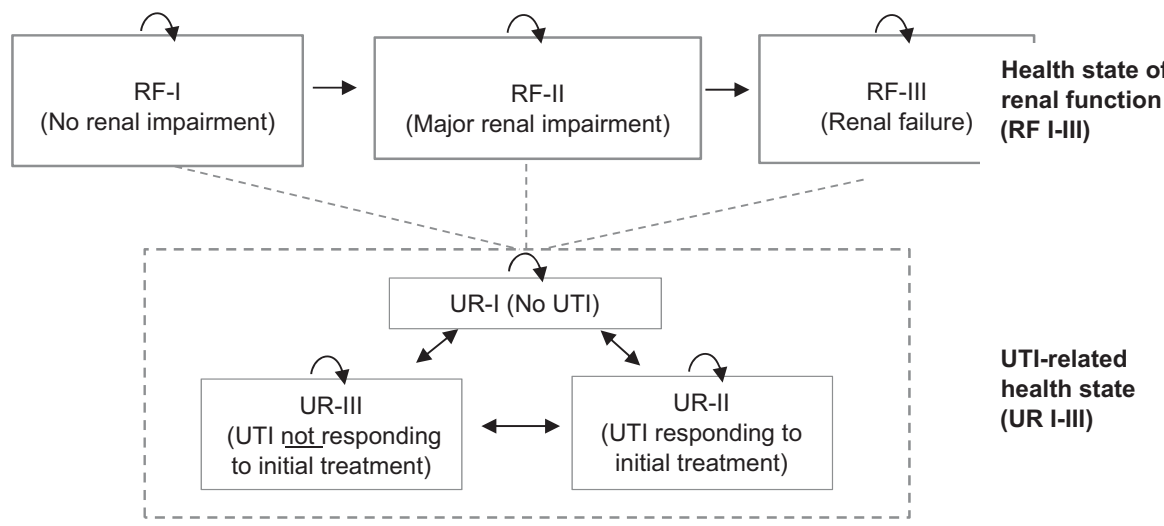

Figure 1 Schematic presentation of the Markov decision model. The three boxes at the upper level present the health state of the renal function (RF). The lower level presents three UTI-related (UR) health states with pre-fixed, equal UTI event rates for all renal function health states. Arrows indicate 'remaining in current state' or 'transition to next state'. Death was possible from all renal- and UTI-related health states.

(renal failure, requiring dialysis or transplantation). Transition represents progression from one health state to the next (patients can only transition to a more severe renal state). At baseline, the whole cohort was assumed to be in health state RF-I.

Within each of the three renal health states, patients had one of three UTI-related (UR) health states: UR-I (no presence of treatment-requiring UTI), UR-II (UTI responding to initial treatment) and UR-III (UTI not responding to initial treatment). It was assumed that across all renal states (RF I-III), patients would have equal rates of UTI-related health states. Death was possible from all renal and UTI-related health states (see Supplementary Material for additional description of the model). Owing to the complexity of the model, the limited data available and the recommendation from the expert panel, it was decided to focus on UTI and renal complications, as illustrated in Figure 1.

\section{Data set}

The objective was to obtain a data set from randomised, controlled trials with adult SCI participants using either HC or UC intermittent self-catheterisation. A comprehensive systematic literature review and cost-effectiveness analysis by Bermingham et al. ${ }^{6}$ identified five studies evaluating HC catheters compared with UC catheters. Only two ${ }^{9,10}$ of these studies included solely adult SCI patients. Subsequently, one further study, investigating HC versus UC in adult SCI patients, has been published; ${ }^{7}$ this was not included in the Bermingham analysis.

Three different scenarios were considered in relation to UTI event rates. For the primary analysis (Table 1a), UTI event rates were based on hospital records, as these data are considered the most valid because they are obtained in a controlled environment. The data set for UTI event rates in a hospital setting included a total of 175 participants using UC catheters and 165 using HC catheters (Table 1a). The secondary data set (Table $1 \mathrm{~b}$ ) included only long-term community UTI event rates from patients with an IC history of $>6$ months. The third data set (Table 1c) combined data from both hospital and community settings. The second and third data sets were used for the sensitivity analysis.

On the basis of selected studies, the primary data set relates to a hypothetical cohort suffering from chronic urinary retention $(80 \%$ male, $20 \%$ female, average age 36 years). At baseline all participants were assumed to be in health state UR-I (No UTI), with a monthly risk of developing treatment-requiring UTI (UR-II; UTI responding to initial treatment) calculated to a weighted average of $64 \%$ for UC catheters. For HC catheters a reduction of $21 \%$ was applied (Table 1a). The evidence and sources for risk estimation for transition to the other renal and UTI-related health states are described in the Supplementary Material (Supplementary Table A), along with other key input parameters and assumptions.

\section{Costs}

Lubrication cost for UC catheters was obtained from the latest version of the NICE clinical guideline on infections, ${ }^{3}$ and daily catheter acquisition costs were obtained from the national drug tariff, ${ }^{11}$ assuming five catheterisations per day according to the European Association of Urology recommendation. ${ }^{12}$ Additional treatment-related adverse event costs were derived using a clinically validated treatment pathway, based on those reported in the NICE clinical guideline on infection ${ }^{3}$ as well as from national reference costs, as appropriate. ${ }^{13}$ Nonprocedural costs were estimated based on the appropriate national database. ${ }^{11}$ Costs for the renal health states RF-II (major renal impairment), RF-III (renal failure) and for the UTI-related health state UR-III (UTI not responding to initial treatment), were fixed. Supplementary Table A (supplementary material) provides data on costs in the base case as well as additional information regarding assumptions and calculations.

\section{Model output}

On the basis of the input parameters from Supplementary Table A (Supplementary material) and mortality rate statistics derived from the UK Office for National Statistics ${ }^{14}$ (see Mortality section in Supplementary Material), the model predicts life expectancy for patients using two different intermittent catheter types, based on UTI event rates in a hospital setting. Further results from the model are expressed as incremental cost-effectiveness ratios. Incremental cost-effectiveness ratios are calculated by dividing the difference in costs associated with the two treatments by the difference in benefits expressed as quality-adjusted life years (QALYs) gained, life-years gained, and number of UTI events avoided. A cost-effectiveness plane was applied to illustrate the relation between incremental cost and QALYs gained (see cost-effectiveness plane section in Supplementary Material). A UK National Health Service perspective was used and a discount rate of $3.5 \%$ was applied for costs and benefits. ${ }^{15}$ Results are calculated in accordance with a probabilistic sensitivity analysis, and are considered cost-effective within a $£ 20000 /$ QALY$\mathfrak{E} 30$ 000/QALY range as recommended by NICE. ${ }^{15}$

\section{Sensitivity analysis}

A deterministic one-way sensitivity analysis of selected variables (Supplementary Table B,Supplementary Material) was performed in order to assess the impact of variations and assumptions on changes in the ICER. A scenario analysis was chosen in order to evaluate both best- and worst-case for the variables.

\section{RESULTS}

The model predicts that a 36-year-old SCI patient with chronic urinary retention using UC catheters is expected to live for an additional 22.5 years (95\% credible interval (CrI) 20.7-24.3), which increases to 23.9 years (95\% CrI 21.8-25.9) when using $\mathrm{HC}$ catheters. 
Table 1 Urinary tract infection (UTI) event rates

\begin{tabular}{|c|c|c|c|c|c|}
\hline Catheter types & Source & Patients & $\begin{array}{c}\text { Mean } \\
\text { months }\end{array}$ & $\begin{array}{c}\text { UTIs } \\
\text { per } \\
\text { month }\end{array}$ & $\begin{array}{c}\text { Weighted } \\
\text { mean }\end{array}$ \\
\hline
\end{tabular}

\begin{tabular}{|c|c|c|c|c|c|}
\hline \multirow{4}{*}{ Uncoated } & C & & & & \\
\hline & Cardenas' & 114 & - & 0.68 & \\
\hline & De Ridder ${ }^{10 a}$ & 61 & 12 & 0.55 & \\
\hline & Total & 175 & & & 0.64 \\
\hline \multirow{3}{*}{$\begin{array}{l}\text { Hydrophilic } \\
\text { coated }\end{array}$} & Cardenas $^{7}$ & 105 & - & 0.54 & \\
\hline & De Ridder ${ }^{10 a}$ & 60 & 12 & 0.44 & \\
\hline & Total & 165 & & & 0.50 \\
\hline $\begin{array}{l}\text { Hydrophilic- } \\
\text { coated } \\
\text { treatment effect }\end{array}$ & & & & & 0.79 \\
\hline UTI reduction & & & & & $21 \%$ \\
\hline \multicolumn{6}{|c|}{ b. UTI rates for long-term community } \\
\hline Uncoated & Cardenas $^{9 b}$ & 23 & 12 & 0.14 & 0.14 \\
\hline $\begin{array}{l}\text { Hydrophilic } \\
\text { coated }\end{array}$ & Cardenas $^{9 \mathrm{~b}}$ & 22 & 12 & 0.06 & 0.06 \\
\hline $\begin{array}{l}\text { Hydrophilic- } \\
\text { coated } \\
\text { treatment effect }\end{array}$ & & & & & 0.47 \\
\hline UTI reduction & & & & & $53 \%$ \\
\hline \multicolumn{6}{|c|}{ c. UTI rates including hospital and community (whole period) } \\
\hline \multirow[t]{4}{*}{ Uncoated } & Cardenas $^{9 \mathrm{~b}}$ & 23 & 12 & 0.14 & \\
\hline & Cardenas $^{7}$ & 114 & - & 0.48 & \\
\hline & De Ridder ${ }^{10 a}$ & 61 & 12 & 0.38 & \\
\hline & Total & 198 & & & 0.41 \\
\hline \multirow{4}{*}{$\begin{array}{l}\text { Hydrophilic- } \\
\text { coated }\end{array}$} & Cardenas $^{9 \mathrm{~b}}$ & 22 & 12 & 0.06 & \\
\hline & Cardenas $^{7}$ & 105 & - & 0.48 & \\
\hline & De Ridder ${ }^{10 a}$ & 60 & 12 & 0.34 & \\
\hline & Total & 187 & & & 0.39 \\
\hline $\begin{array}{l}\text { Hydrophilic- } \\
\text { coated } \\
\text { treatment effect }\end{array}$ & & & & & 0.90 \\
\hline UTI reduction & & & & & $10 \%$ \\
\hline
\end{tabular}

Abbreviation: UTI, urinary tract infection.

a Numbers was derived from study report LAH 00.01.6.31 IV, ITT population.

bTotal antibiotic treatment episodes divided by 12 .

For the general UK population an equivalent life expectancy would be 44.4 years. ${ }^{14}$ The probabilistic costs and benefits of each intervention, and all incremental cost-effectiveness ratios, are presented in Table 2, and the cost-effectiveness plane is shown in Figure A of Supplementary Material. At an incremental cost of $£ 2100$ per patient, HC catheters confer 0.35 QALYs and 0.64 discounted life-years gained, resulting in an ICER of $\mathfrak{E} 6100$ per QALY gained and $\mathfrak{E} 3300$ per lifeyears gained per patient. $^{15}$

Estimated UTI events (undiscounted) for a patient's lifetime were reduced by $16 \%$ using HC catheters (143 UTI events) compared with UC catheters (170 UTI events; Table 2). This gives an ICER of $£ 79$ per overall UTI avoided per patient.

The one-way sensitivity analysis showed that $\mathrm{HC}$ catheters were the dominant technology (offering increased benefits at lower cost) when the UTI event rate in the long-term community setting was reduced from 0.14 for UC catheters to 0.06 for $\mathrm{HC}$ catheters (Table $1 \mathrm{~b}$ ). When assessing both hospital and community periods, a UTI reduction rate of $10 \%$ (Table 1c) resulted in an ICER of $£ 16400$ per QALY gained (Supplementary Table B Supplementary Material). When the UTI reduction rate of $21 \%$ found in the hospital period (Table 1a) for HC catheters was applied to the lowest published UTI event rate of 0.06 for HC catheters (Table 1b), the ICER was estimated at $£ 26440$ per QALY gained, which is still within the UK threshold of being costeffective. When the incidence of UTI increases, the cost-effectiveness of HC catheters will also increase. The model was largely insensitive to changes in the remaining parameters (Supplementary Table B; Supplementary Material); results were all within the threshold considered cost-effective by NICE.

\section{DISCUSSION}

A probabilistic decision analysis was conducted in order to investigate the cost-effectiveness of $\mathrm{HC}$ catheters compared with UC catheters. Using a lifetime perspective, $\mathrm{HC}$ catheters were estimated to be a costeffective solution when compared with UC catheters, with an ICER of

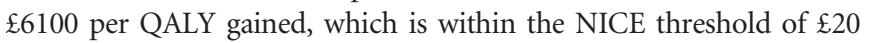
000-£30 000 per QALY.

The findings are different from the results published by Bermingham et al., ${ }^{6}$ which estimated that over lifetime, gel-reservoir catheters were associated with an average gain of 0.52 QALYs per patient compared with clean UC catheters; however, the ICER per QALY in that study exceeded the NICE recommended threshold. Bermingham et al. ${ }^{6}$ did not include the long-term sequelae of UTI but only 'acute' management issues, and their analysis did not include the latest and largest randomised controlled trial comparing single use of UC and HC catheters. ${ }^{7}$ In addition, the included studies had a different study population, and did not exclude to single use of either HC or UC catheters. The results showing preference of gel-reservoir catheters over HC catheters were highly affected by a single-centre crossover study with 18 patients by Giannantoni et al. ${ }^{16}$ where the definition of UTI was not clear. The conclusions from Bermingham et al. ${ }^{6}$ were also modified to a broader perspective, owing to limitations and gaps in the evidence base, recommending that patients should be offered a choice between $\mathrm{HC}$ and gel-reservoir catheters.

The 21\% difference in UTI incidence rates between the HC and UC catheters applied in the present analysis was based on data collected in hospital settings (Table 1a), whereas the $10 \%$ difference used for the sensitivity analysis used data covering both hospital and community periods (Table 1c). The 53\% difference used for the upper limit was based on a purely community setting, where participants on average had used IC for $>10$ years (Table $1 \mathrm{~b}$ ). Hospital data were chosen as the primary data set, as UTI event rates were considered more valid when obtained in a controlled environment, whereas data collected after hospital discharge are based upon voluntary reporting from SCI patients. A revised analysis performed by Cochrane (oral presentation, ICS, Rio, 2014), including both community and hospital data, ${ }^{9,10}$ showed a $21 \%$ reduction in patients experiencing treatment-requiring UTI when using HC catheters compared with UC catheters $(P=0.04)$. In the light of this, the $10 \%$ reduction rate applied in our sensitivity analysis for the hospital and community period may be too conservative.

The cost prices applied in our analysis are also conservative estimates compared with the cost prices used by Bermingham et al., ${ }^{6}$ who used $\mathfrak{£} 1.19$ for UC catheters and $\mathfrak{E} 1.42$ for HC catheters

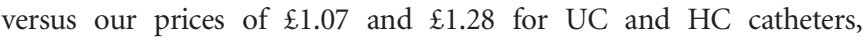
respectively. If the prices applied in Bermingham et al. ${ }^{6}$ were used in 
Table 2 Probabilistic cost effectiveness results (base case)

\begin{tabular}{|c|c|c|c|c|}
\hline & Costs $^{\mathrm{a}}$ & $Q A L Y S^{\mathrm{a}}$ & $L Y G^{a}$ & UTI events ${ }^{b}$ \\
\hline Uncoated catheters & $\begin{array}{c}£ 59000 \\
(£ 54900 ; £ 64100)\end{array}$ & $\begin{array}{c}6.58 \\
(5.87 ; 7.30)\end{array}$ & $\begin{array}{c}14.75 \\
(13.96 ; 15.53)\end{array}$ & $\begin{array}{c}169.98 \\
(142.15 ; 198.73)\end{array}$ \\
\hline Incremental values & $\begin{array}{c}£ 2100 \\
(1500 ; 2800)\end{array}$ & $\begin{array}{c}0.35 \\
(0.34 ; 0.37)\end{array}$ & $\begin{array}{c}0.64 \\
(0.51 ; 0.68)\end{array}$ & $\begin{array}{c}-26.48 \\
(-29.76 ;-21.86)\end{array}$ \\
\hline
\end{tabular}

Abbreviations: LYG, life-years gained; UTI, urinary tract infection; QALY, quality-adjusted life years.

acosts, QALYS, and LYG presented as discounted mean and $95 \%$ credible interval.

bUTI events undiscounted.

our analysis, the difference would have been even more favourable for HC catheters.

\section{Limitations and strengths}

The most pronounced limiting factor for this cost-effectiveness model is the lack of publications on the use of $\mathrm{HC}$ and UC and the associated complications. In order to compensate for this, the expert panel provided input where data were unavailable. Three studies were considered eligible, as only adult SCI patients using single-use UC or HC catheters were included. Only two of the studies were included in the primary data set to establish the UTI event rates based on hospital settings used in the model. While different types of catheters are available, the model was predicated to evaluate the costeffectiveness of two types of single-use catheters, HC and UC, because they are the most frequently used catheters in UK. ${ }^{11}$ Other equally valid decision problems on less frequently used catheters could have been addressed; for example, gel-reservoir, however, published data on their use are limited. In general, management of UTIs will always be subject to local decisions and practicalities, however, this should not influence the differences between $\mathrm{HC}$ and UC catheters per se. Nevertheless, it is clear that with the introduction of aseptic (European Association of Urology Nurses definition) IC the UTI complication rate is reduced markedly. ${ }^{17}$

The model assumes five catheterisations daily, based on the recommendation by European Association of Urology. ${ }^{12}$ In case of noncompliance with this the risk of UTI can increase, and hence the risk for further long-term sequelae and consequent economic burdens increases as well. The risk of acquiring sequelae after UTI is based on literature data, mostly collected in retrospective analyses of existing data in hospital charts; this is an uncertainty that limits the model when used in a community setting.

The life expectancy estimated by the model is lower than the actual life expectancy found for SCI patients. However, data used for the estimations are based on a hospital setting, where the incidence of UTIs is nearly three-fold that found in the community setting. However, this should not affect the incremental difference found.

In conclusion, from a UK perspective and considering the premises of the model assumptions, the use of $\mathrm{HC}$ catheters for IC in SCI patients is highly cost effective. The outcome is consistent irrespective of whether data are collected in hospital or community settings. The model is applicable for other countries as the data are based on international literature; however the use of country-specific costs and health care system parameters is recommended for optimal adaption.

\section{DATA ARCHIVING}

There were no data to deposit.

\section{CONFLICT OF INTEREST}

SJM, JFC and DAS work for an international consultancy company, which receives payment from a number of large pharmaceutical/medical companies, including Coloplast. However, none of the authors have received any personal remuneration of any kind from Coloplast for any work, including the materials presented in this paper. AK's work is supported by Canadian Institute for Health Research, C. Nielsen Foundation, Rick Hansen Institute, Canadian Foundation for Innovation, Allergan, Wellspect, Purdue. The remaining authors declare no potential conflict of interest.

\section{ACKNOWLEDGEMENTS}

We thank Professor Dr Jürgen Pannek (Schweizer Paraplegiker-Zentrum, Nottwil) for his expert advice during the construction of the model. Mia Buus Andersen and Zenia M. Størling (Coloplast A/S) provided critical scientific review of the manuscript. The study was supported by research funding from Coloplast A/S. However, no restrictions were placed on the design of the study, the choice of included data sources or the presentation of results.

1 Apparelyzed. Availabe at www.apparelyzed.com/statistics.html. Accessed on 28 November 2014.

2 Singh M, Perkash I, Bodner DR. Urologic Management in spinal cord injury. In: Lin VW (ed.) Spinal Cord Medicine, Principles and Practice, 2nd edn. Demos Medical Publishing: 2010.

3 NICE (National Institute for Health and Care Excellence) Infection: prevention and control of healthcare-associated infections in primary and community care. Available at http://www.nice.org.uk/guidance/cg139/chapter/guidance. 2012.

4 Vahr S, Cobussen-Boekhorst H, Eikenboom J, Geng V, Holroyd S, Lester M et al. Catheterisation. Urethral Intermittent in Adults: Dilatation, Urethral Intermittent in Adults. European Association of Urology Nurses (EAUN): Arnhem, The Netherlands, 2013, 96pp.

5 Prieto JA, Murphy C, Moore KN, Fader MJ. Intermittent catheterisation for long-term bladder management (abridged Cochrane Review). Neurourol Urodyn 2015; doi:10.1002/nau.22792.

6 Bermingham SL, Hodgkinson S, Wright S, Hayter E, Spinks J, Pellowe C. Intermittent self catheterisation with hydrophilic, gel reservoir, and non-coated catheters: a systematic review and cost effectiveness analysis. BMJ 2013; 346: e8639.

7 Cardenas DD, Moore KN, Dannels-McClure A, Scelza WM, Graves DE, Brooks M et al. Intermittent catheterization with a hydrophilic-coated catheter delays urinary tract infections in acute spinal cord injury: a prospective, randomized, multicenter trial. $P M R$ 2011; 3: 408-417.

8 Briggs A, Claxton K, Sculpher M. Decision Modelling for Health Economic Evaluation. Oxford University Press. 2006.

9 Cardenas DD, Hoffman JM. Hydrophilic catheters versus noncoated catheters for reducing the incidence of urinary tract infections: a randomized controlled trial. Arch Phys Med Rehabil. 2009; 90: 1668-1671.

10 De Ridder DJ, Everaert K, Fernandez LG, Valero JV, Duran AB, Abrisqueta ML et al. Intermittent catheterisation with hydrophilic-coated catheters (SpeediCath) reduces the risk of clinical urinary tract infection in spinal cord injured patients: a prospective randomised parallel comparative trial. Eur Urol 2005; 48: 991-995. 
11 National Health Service England and Wales NHS Electronic Drug Tariff. Availabe at www.ppa.org.uk/edt/September_2012/mindex.htm. Accessed on February 2012.

12 Pannek J, Blok B, Castro-Diaz D, Del Popolo G, Kramer G, Radziszewski P et al. Guidelines on Neurogenic Lower Urinary Tract Dysfunction. European Association of Urology. Availabe at http://uroweb.org/wp-content/uploads/20_Neurogenic-LUTD_LR.pdf 2013.

13 NHS Reference Costs. Availabe at https:/www.gov.uk/government/uploads/system/ uploads/attachment_data/file/215297/dh_131160.pdf. 2011.

14 UK National Statistics. Gov.UK officail statistics. Availabe at http://www.statistics.gov. uk/hub/index.html. Accessed on August 2012
15 NICE (National Institute for Health and Care Excellence). Guide to the methods of technology appraisal. Availabe at http://www.nice.org.uk/article/pmg9/resources/nonguidance-guide-to-the-methods-of-technology-appraisal-2013-pdf. Accessed on 2013.

16 Giannantoni A, Di Stasi SM, Scivoletto G, Virgili G, Dolci S, Porena M. Intermittent catheterization with a prelubricated catheter in spinal cord injured patients: a prospective randomized crossover study. J Urol 2001; 166: 130-133.

17 Wyndaele JJ. Complications of intermittent catheterization: their prevention and treatment. Spinal Cord 2002; 40: 536-541.

Supplementary Information accompanies this paper on the Spinal Cord website (http://www.nature.com/sc) 